\title{
Debye to non-Debye scaling of the Boson peak dynamics: Critical behavior and local disorder in vitreous germania
}

\author{
M. Zanatta, ${ }^{1,2, a)}$ G. Baldi, ${ }^{3,4}$ S. Caponi, ${ }^{1,5}$ A. Fontana, ${ }^{1,2}$ C. Petrillo, ${ }^{5,6}$ F. Rossi, ${ }^{1}$ \\ and F. Sacchetti ${ }^{5,6}$ \\ ${ }^{1}$ Dipartimento di Fisica, Università di Trento, I-38123 Povo, Trento, Italy \\ ${ }^{2}$ CNR-IPCF, UOS di Roma, clo Università di Roma "La Sapienza," I-00185 Roma, Italy \\ ${ }^{3}$ CNR-IMEM, I-43124 Parma, Italy \\ ${ }^{4}$ Dipartimento di Fisica, Università di Parma, I-43124, Parma, Italy \\ ${ }^{5}$ CNR-IOM clo Dipartimento di Fisica, Università di Perugia, I-06123 Perugia, Italy \\ ${ }^{6}$ Dipartimento di Fisica, Università di Perugia, I-06123 Perugia, Italy
}

(Received 9 August 2011; accepted 10 October 2011; published online 2 November 2011)

\begin{abstract}
We report on the observation of a significant softening of the Boson peak (BP) frequency of vitreous $\mathrm{GeO}_{2}$ above the glass transition temperature. The softening reminds a critical trend, with a transition temperature intermediate between the glass transition temperature, $T_{g}$, and the melting point. The softening of the BP frequency corresponds to a transition from a region dominated by Debye scaling to a non-Debye one. Below $T_{g}$ the density of vibrational states varies according to the modification of the elastic continuum medium. Above $T_{g}$ the relevant softening of the BP modes can be explained by a broadening of the distribution of elastic constants between neighboring atoms, induced by the structural rearrangement. These findings are discussed together with recent experimental and theoretical results on the low frequency vibrations in glasses. (C) 2011 American Institute of Physics. [doi:10.1063/1.3656697]
\end{abstract}

\section{INTRODUCTION}

A universal feature of the dynamics of glasses and supercooled liquids is an excess of vibrational modes above the Debye level. This excess is called boson peak (BP) and it is visible as a broad maximum in the reduced density of vibrational states $g(\omega) / \omega^{2}$. The importance of the BP goes beyond the physics of glasses. Indeed, this feature is common to a broad variety of systems with different chemical and physical properties, from polymers and colloids to biological systems. ${ }^{1-4}$ The BP is an evident fingerprint of the intrinsic disorder present in the system.

A variety of hypothesis has been advanced in the literature to explain the nature of the vibrational modes in the BP frequency range. ${ }^{5-14}$ They can be roughly grouped into two main classes. The first one is based on the soft potential model, ${ }^{5,6}$ which assumes a certain degree of anharmonicity in the interatomic potential, and suggests the BP to be mainly due to quasi-localized excitations, originating from the hybridization of some localized modes with the plane waves. The existence of vibrations with high intensity on only a few atoms seems confirmed in particular colloidal aggregates and in granular materials, whose eigenvectors and eigenvalues can be studied both numerically ${ }^{15,16}$ and experimentally. ${ }^{17}$ However, the connection between the physical properties of these systems and that of real covalent glasses still remains highly speculative.

A second class of models considers the glass as a harmonic solid, whose atoms vibrate around average positions on a disordered network. The disorder breaks the translational

\footnotetext{
a)Electronic mail: zanatta@science.unitn.it.
}

invariance of the associated crystalline lattice and gives rise to a distribution of elastic constants. In this second group of models the most convincing interpretation indicates the BP as the counterpart of the first van Hove singularity of the corresponding crystal. ${ }^{8}$ Evidence on the validity of this interpretation has been obtained both numerically ${ }^{18,19}$ and from inelastic x-ray and neutron scattering experiments. ${ }^{20-23}$ The glass can sustain acoustic modes up to the border of a pseudo-Brillouin zone and the BP is located around the frequency of the transverse acoustic excitation at the zone border. Very recently, Chumakov and co-workers ${ }^{24}$ have given further strength to this interpretation, showing how the BP modes of a silicate glass shift towards the frequency of the transverse acoustic excitation of the crystal as the density is increased towards that of the crystalline phase.

An effective approach to gather information on the nature of the boson peak is the investigation of its evolution as a function of thermodynamical parameters, such as temperature ${ }^{25-27}$ and pressure. ${ }^{4,28-30}$ In general, an increase of the system density gives rise to a shift of the BP towards higher frequencies and to a simultaneous decrease of its intensity. This has been observed also in a permanently densified glass, ${ }^{24,31-33}$ and during a chemical vitrification process, where the system evolves with the reaction time. ${ }^{34}$ All these studies have shown that the BP intensity variation is only due to its frequency shift. This finding is highlighted by the existence of a master curve that has also been theoretically predicted. ${ }^{9}$

The need for a satisfactory explanation of the BP evolution encompassed in a universal scaling law started with the investigation of its position. A first guess is obtained treating the system as an elastic medium evolving as a function 
of the external parameters and characterized by the Debye frequency $\omega_{D}$. This approach provides a good scaling law only in some systems, ${ }^{24,26,31,34}$ whereas it does not work in many other cases. ${ }^{4,25,27,28,32,33}$ These conflicting results are still waiting for a global explanation. As a matter of fact, differences between multiple case-studies do not have to be considered as anomalies but most likely as different aspects of the same disorder related mechanism.

In this paper we present a detailed study of the temperature evolution of the BP vibrational dynamics of vitreous germania $\left(\mathrm{v}-\mathrm{GeO}_{2}\right)$. Like $\mathrm{v}-\mathrm{SiO}_{2}, \mathrm{v}-\mathrm{GeO}_{2}$ is a covalent oxide glass and both are prototypes of the strong network forming glasses. This class of glasses is well known for structural and dynamical stability, which is maintained at temperatures even higher than the melting temperature $T_{m}$. In the case of v- $\mathrm{GeO}_{2}$ we have a glass transition temperature $T_{g} \simeq 800 \mathrm{~K}$ and $T_{m}=1370 \mathrm{~K}$. Collecting Raman spectra in a temperature range from $15 \mathrm{~K}$ to $1200 \mathrm{~K}$, the boson peak position $\omega_{\mathrm{BP}}$ shows an abrupt decrease followed by a plateau. The shape of this softening reminds a critical trend occurring above $T_{g}$ and marking a transition from a Debye scaling to a non-Debye one. The Debye frequency $\omega_{D}$ is computed in the same $T$ region using data from Refs. 35-39.

The existence of this crossover suggests the presence of at least another mechanism ruling the BP shift, indicating vitreous $\mathrm{GeO}_{2}$ as the perfect test bench to identify the contribution which dominates in the supercooled liquid region. To unveil the origin of this crossover we investigate also the evolution of the static structure factor in the same temperature region obtaining a detailed picture of the system evolution. We consider two mechanisms associated to the BP shift with temperature. One is the pseudo-Brillouin zone boundary modification, following the approach of Refs. 8, 19, and 24. The other one is the spread in the shear modulus distribution, as obtained considering the disordered elastic medium model of Refs. 10-12. Both of them highlight different aspects of the same process, which gives rise to the fluctuation of the force constants and to the evolution of the first sharp diffraction peak (FSDP).

\section{EXPERIMENTS AND RESULTS}

Vitreous germania samples have been prepared starting from Aldrich germanium (IV) oxide crystalline powder (purity higher than $99.999 \%$ ). The powder was melted in an alumina crucible at about $1900 \mathrm{~K}$, and then quenched in air.

Raman measurements were performed using a JobinYvon U1000 double monochromator in $90^{\circ}$ scattering geometry both in $\mathrm{HV}$ and $\mathrm{VV}$ polarization. As a light source, we used the $5145 \AA$ line of an argon ions laser. Raman spectra were collected over a wide range of frequency from -650 to $1660 \mathrm{~cm}^{-1}$. For the low frequency spectra, the resolution was fixed at about $2 \mathrm{~cm}^{-1}$. The wide frequency range is necessary in order to properly subtract the background. Special care has been devoted to avoid crystallization of the sample when entering the supercooled region, by looking at the Raman spectrum for signatures of crystal formation.

In Fig. 1, the depolarized (HV) reduced Raman intensity $I^{r e d}(\omega)$ is reported for selected temperatures. The formal con-

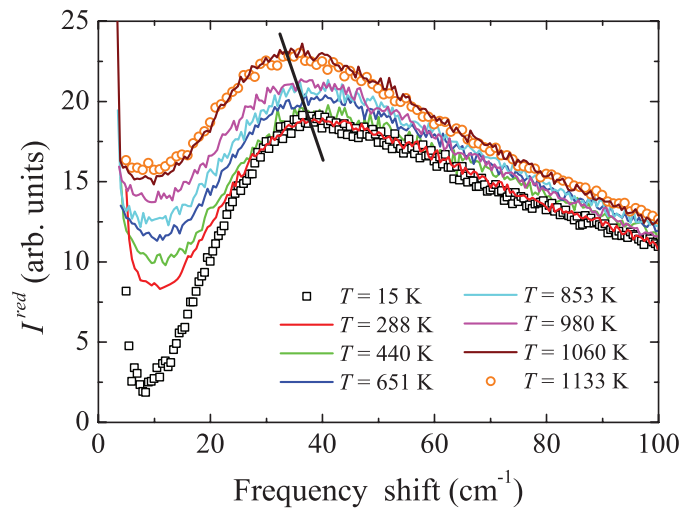

FIG. 1. Reduced Raman HV spectra for selected temperatures, the thick line is a guide for the eyes and it indicates the BP evolution; temperature values are reported in the legend. Spectra are normalized to the area of the $840 \mathrm{~cm}^{-1}$ molecular peak, as detailed in Ref. 37. Notice the continuous line at $T=288 \mathrm{~K}$ which shows no change of the Raman spectrum with respect to $T=15 \mathrm{~K}$ and the continuous line at $T=1060 \mathrm{~K}$ which again shows no further changes at higher temperatures.

nection between $I^{r e d}(\omega)$ and $g(\omega)$ is far from being simple. ${ }^{40}$ In the following we use the effective relation ${ }^{41}$

$$
I^{r e d}(\omega)=\frac{I^{e x p}}{[n(\omega, T)+1] \omega}=C(\omega) \frac{g(\omega)}{\omega^{2}},
$$

where $I^{e x p}$ is the experimental Raman intensity, $n(\omega, T)$ is the Bose population factor, and $C(\omega)$ is the light-vibrations coupling function. Besides the boson peak, the low frequency region $\left(\omega \lesssim 10 \mathrm{~cm}^{-1}\right)$ is characterized by the rise of $T$-dependent quasielastic scattering (QES). This contribution does not affect the position and the intensity of the BP. For $T$ $<300 \mathrm{~K}$, apart for the QES rise, the spectra do not show any change. Above room temperature, the BP starts to shift downwards and it increases its intensity. This trend is highlighted by a thick line in Fig. 1. Going well above $T_{g}$, the BP does not evolve anymore, i.e., the peak frequency $\omega_{\mathrm{BP}}$ and its intensity become constant.

Neutron diffraction experiments were carried out at the 7C2 two axis spectrometer, located on the hot source of the reactor Orphée of the Laboratoire Leon Brillouin (LLB, Saclay, France). The incident wavelength $\lambda=0.729 \AA$ was chosen to access a wide momentum transfer range between 0.4 and $15 \AA^{-1}$ and to minimize the inelasticity corrections. The experimental intensity was reduced using Monte Carlo simulation to estimate the multiple scattering, the cell contribution, and the transmission coefficients. ${ }^{42,43}$ Spectra were acquired as a function of $T$, from room temperature to $1000 \mathrm{~K}$. Accessing the supercooled liquid region above $1000 \mathrm{~K}$, besides the typical amorphous pattern, Bragg peaks appeared, thus indicating the start of crystal's nucleation processes. Raman spectra were not affected by this problem since experiments lasted considerably less and the scattering volume was smaller than that in neutron scattering experiments.

From the structural point of view glasses, and in particular network forming glasses, are characterized by a rather well defined short range order, dominated by structural units which roughly correspond to the crystalline ones. ${ }^{44}$ In the case of vitreous germania the building block is the $\mathrm{Ge}\left(\mathrm{O}_{1 / 2}\right)_{4}$ tetrahedra. Beyond this short range, topological disorder 


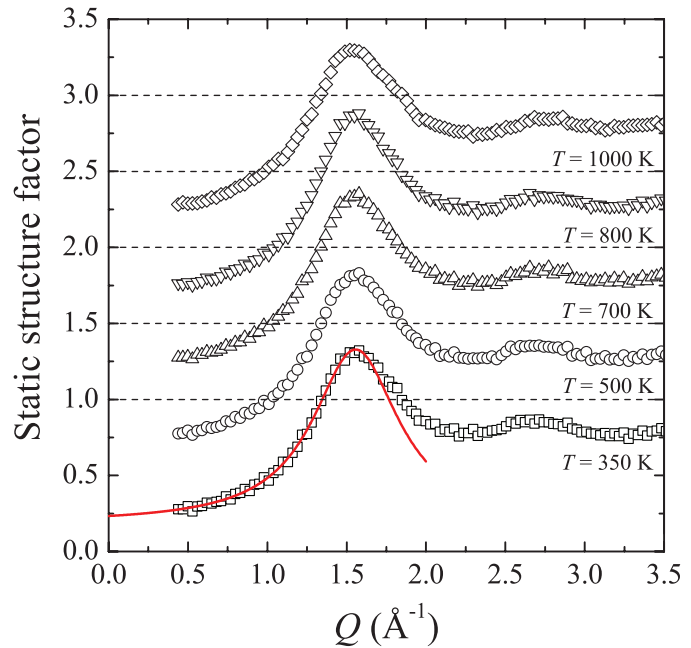

FIG. 2. FSDP at selected temperatures; spectra are reported by shifting progressively data by 0.5 in the $y$ axis. The continuous line is an example of the fits obtained using a Lorentzian function, see text.

comes into play, even if for distances corresponding to some structural units a degree of ordering is still present. This region originates the low momentum $Q$ part of the static structure factor $S(Q)$, which is reported in Fig. 2 for selected temperatures. In particular, we focus our attention to the so called FSDP whose position $Q_{1}$ is thought to reflect an intermediate range ordering. ${ }^{45}$ To evaluate $Q_{1}$, the FSDP has been fitted with a Lorentzian function considering only the lower $Q$ side of the peak in order to avoid contributions from the subsequent diffraction structures. ${ }^{46}$

\section{DISCUSSION}

We start the analysis of the present data by considering the BP spectral shape as a function of a scaled frequency $v=\omega / \omega_{\mathrm{BP}}$ and performing the variable transformation $g(v) d v=g(\omega) d \omega$. Assuming that in the BP frequency region $C(\omega) \sim \omega$ (Refs. $47-50$ ) and $T$ independent, ${ }^{47,49}$ the intensity as a function of $v$ can be written as

$$
I(v)=I^{r e d}(\omega) \times \omega_{\mathrm{BP}}^{2} .
$$

The BP positions are obtained by fitting a log-normal function to the Raman data. The $I(v)$ spectra are reported in Fig. 3 where it is shown that spectra corresponding to different temperatures collapse into a single master curve without any adjustable parameter. The existence of this master curve shows that the BP evolution can be explained by considering only the behavior of $\omega_{\mathrm{BP}} .^{25,33}$

In order to compare the BP evolution to that of the elastic medium, we first derive $\omega_{D}$ versus temperature from the Brillouin longitudinal and transverse sound velocities ${ }^{36}$ and the density values of Ref. 38 and 39. Since the sound velocity and the density do not show significant changes, no anharmonic behavior is expected in the whole investigated temperature range. The comparison between the temperature evolution of $\omega_{\mathrm{BP}}$ and that of $\omega_{D}$ is reported in Fig. 4(a). Both datasets are normalized to their low temperature value. For $T<800 \mathrm{~K}$, $\omega_{\mathrm{BP}}$ and $\omega_{D}$ exhibit a similar behavior in agreement with pre-

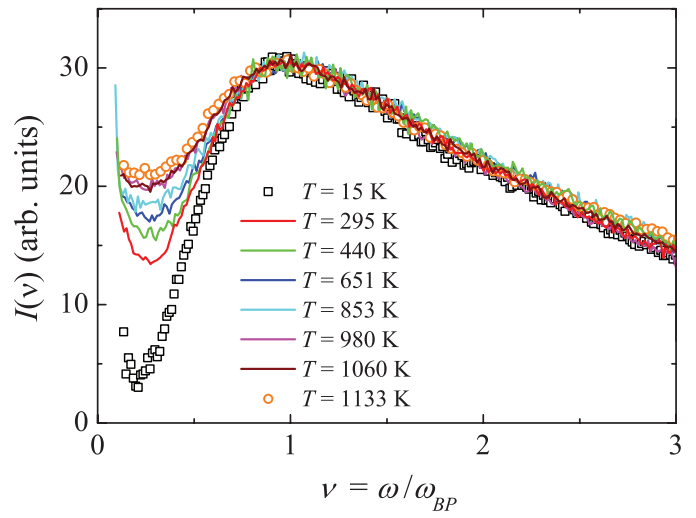

FIG. 3. Master curve obtained by squeezing $I^{\text {red }}(\omega)$ as described in the text at selected temperatures; the BP positions are obtained by fitting a log-normal function to the Raman data. Notice the perfect agreement of the BP obtained with no adjustable parameter. The existence of this master curve demonstrates that the increase of the BP intensity is only an apparent effect due to its shift as a function of $T$.

vious findings, indicating the $\omega_{\mathrm{BP}}$ shift to be accounted for by the $\omega_{D}$ change. ${ }^{26,31}$ When $T$ exceeds $800 \mathrm{~K}, \omega_{\mathrm{BP}}$ shows an abrupt decrease $(\simeq 15 \%)$ which cannot be explained by the change of $\omega_{D}$ (see Fig. 4(a)). In this temperature range a new process, affecting the BP behavior, is taking place. Finally for $T>1000 \mathrm{~K}$, well inside the supercooled liquid region, $\omega_{\mathrm{BP}}$ remains constant.

Since the behavior of $\omega_{\mathrm{BP}}$ reminds a critical trend, we tentatively fit its evolution using a mean field approximation

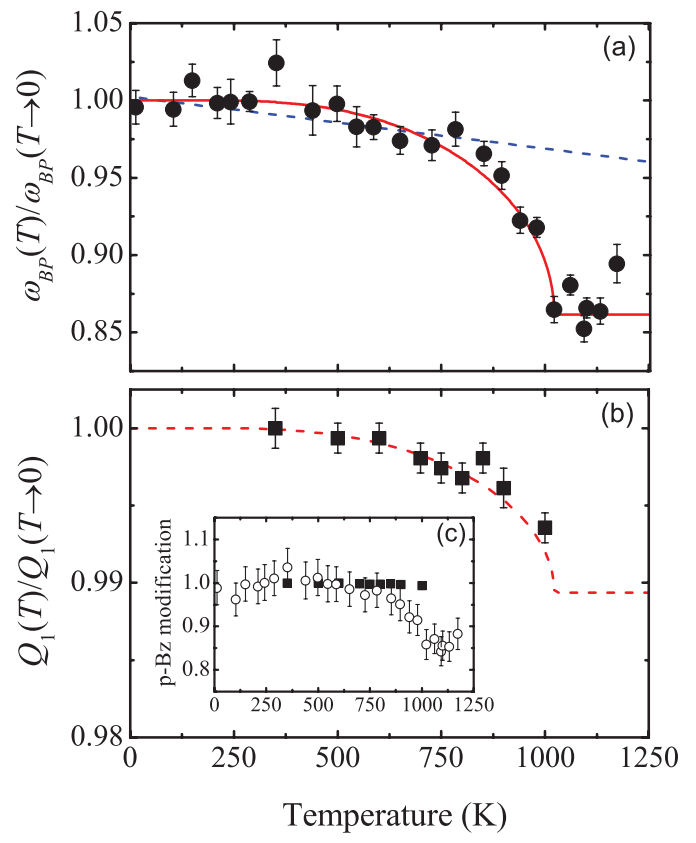

FIG. 4. (a) $\omega_{\mathrm{BP}}(T) / \omega_{\mathrm{BP}}(T \rightarrow 0 \mathrm{~K})$ (black dots), $\omega_{D}(T)$ (dashed line), and mean field approximation (full line, see text) as a function of temperature. The data are normalized to their low temperature values, $\omega_{B P}^{n o r m}=40.3 \mathrm{~cm}^{-1}$ and $\omega_{D}^{\text {norm }}=212 \mathrm{~cm}^{-1}$. (b) $Q_{1}$ (black squares) and fit with a mean field function with the same parameters as in (a) times a proportionality factor (dashed line). (c) Comparison between $Q_{1} / 2$, one-half of the FSDP position (black squares), and $Q_{0}$ (open circles), the pseudo-Brillouin zone $(\mathrm{p}-\mathrm{Bz})$ boundary modification, required to account for the observed BP shift in the framework of the model proposed in Ref. 24 (see text for details). Each dataset is normalized to its lowest temperature value, $Q_{1}^{\text {norm }}=1.55 \AA^{-1}$ and $Q_{0}^{\text {norm }}=0.52 \AA^{-1}$. 
in the following spin $1 / 2$ form: ${ }^{51}$

$$
\begin{gathered}
\omega_{\mathrm{BP}}=\left(\omega_{0}-\omega_{\infty}\right) S(T)+\omega_{\infty}, \\
S(T)=\tanh \left[\frac{S(T) T_{\mathrm{BP}}}{T}\right],
\end{gathered}
$$

where $\omega_{0}$ is $\omega_{\mathrm{BP}}$ at $T=15 \mathrm{~K}, \omega_{\infty}$ is the constant value observed above $T_{\mathrm{BP}}$, and $S(T)$ is the order parameter of the mean field approximation characterized by the critical temperature $T_{\mathrm{BP}}$. As it is seen in Fig. 4(a), the above mean field approximation, plotted as a continuous line, provides a very good fit to the experimental data with a transition temperature $T_{\mathrm{BP}}=(1020 \pm 20) \mathrm{K}$. Interestingly, the temperature $T_{\mathrm{BP}}$ lies between the glass transition temperature, $T_{g} \simeq 800 \mathrm{~K}$, and the melting point, $T_{m} \simeq 1370 \mathrm{~K}$.

In the glassy state, $T<T_{g}$, the BP frequency presents a temperature dependence close to that of the Debye frequency $\omega_{D}(T)$. In the Debye limit, the system is considered as a continuum, whose vibrations are plane waves of longitudinal and transverse polarization. For $T<T_{g}$ the system is thus well described as an elastic medium, whose vibrational spectrum varies with temperature according to the variation of a few macroscopic parameters, the sound velocity and the density. The non-Debye trend of the BP above $T_{g}$ requires, on the contrary, a microscopic modification of the structure or of the elasticity of the glass. A structural variation can be indeed observed looking at the temperature evolution of the wavevector position, $Q_{1}$, of the first sharp diffraction peak. $Q_{1}$ is affected by a small, $\sim 1 \%$, but still well detectable softening as the temperature is increased above $T_{g}$. This trend can be well fitted by the same mean field curve used to describe the BP evolution times a proportionality factor, as shown in Fig. 4(b). The good agreement between the curve and the data highlights further the connection between the BP variation and this structural rearrangement.

It is worth noting, however, that the variation of the FSDP is very small $(\simeq 1 \%)$ compared to the large $(\simeq 15 \%)$ change in the BP frequency. The FSDP can, in fact, be viewed as a broadened Bragg peak. In a crystal, the first Bragg peak corresponds to a reciprocal lattice vector and thus approximately to twice the Brillouin zone boundary. ${ }^{52}$ Following this argument, $Q_{1} / 2$ is a good estimation of the pseudo-Brillouin zone border. On the other hand, if the BP arises from the bending of the transverse acoustic modes, the zone border should be roughly equal to

$$
Q_{0}=\frac{\pi^{2} c \omega_{\mathrm{BP}}}{v_{T}},
$$

where $v_{T}$ is the transverse sound velocity, $c$ is the speed of light in vacuum, and $\omega_{\mathrm{BP}}$ is the boson peak frequency in wavenumber units. Such a definition of $Q_{0}$ assumes the transverse modes to disperse as a sine function up to the zone border. Figure 4(c) shows the temperature evolution of the two quantities $Q_{0}$ and $Q_{1} / 2$, both normalized to their low temperature values. Clearly, the small variation of the pseudoBrillouin zone boundary, $Q_{1} / 2$, cannot account for the large variation of $\omega_{\mathrm{BP}}$, which would require a similarly large change of the zone boundary, as indicated by $Q_{0}$.

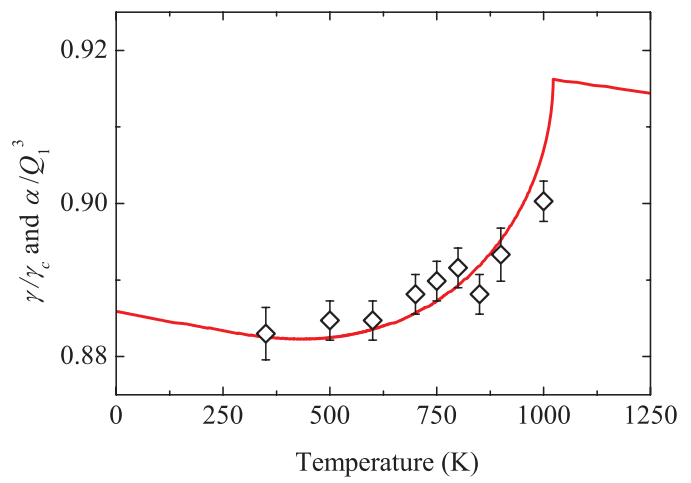

FIG. 5. Temperature evolution of the disorder parameter $\gamma / \gamma_{c}$ (full line), obtained inverting the function $\omega_{\mathrm{BP}}^{t h}(\gamma)$. The critical disorder parameter $\gamma_{c}$ is defined within the Shirmacher model. ${ }^{10-12}$ The input parameter is the normalized boson peak position $\omega_{\mathrm{BP}} / \omega_{D}$. Black diamonds represent $1 / Q_{1}^{3}$ times a proportionality factor $\alpha$.

This apparent contradiction can be solved considering that a structural variation can be accompanied by a change in the distribution of the elastic constants between neighboring atoms. The presence in the glass of a distribution of elastic constants is the central ingredient of the Shirmacher and coworkers model. ${ }^{11}$ This is a theory for the vibrational excitations in a disordered elastic medium, based on the assumption that the shear modulus exhibits random spatial fluctuations, whose variance is proportional to a disordered parameter $\gamma$. In this model the density of vibrational states is derived from the self-energy given by ${ }^{10-12}$

$$
\Sigma(\omega)=\gamma \sum_{k<k_{D}}\left[\chi_{L}(k, \omega)+\chi_{T}(k, \omega)\right],
$$

where $\chi_{L}(k, \omega)$ and $\chi_{T}(k, \omega)$ are the longitudinal and transverse dynamic susceptibilities, which are themselves function of $\Sigma$, and $k_{D}$ is the Debye wave vector. Having computed the frequency dependence of the self-energy from the set of self-consistent equations defining the model (Eq. (1) of Ref. 11), we derive the density of vibrational states as a function of $\gamma$. In this theory the reduced density of vibrational states, $g(\omega) / \omega^{2}$, shows a BP maximum whose position $\omega_{\mathrm{BP}}^{t h}$ depends upon the value of the disorder parameter $\gamma$. The function $\omega_{\mathrm{BP}}^{t h}(\gamma)$ is a monotonically decreasing function of $\gamma$ that can be computed numerically from the above equation. Moreover, $\omega_{\mathrm{BP}}^{t h}$ exhibits a critical-like transition towards zero frequency when $\gamma$ approaches a critical value $\gamma_{c}$.

In order to compare the model with the data we have inverted the function $\omega_{\mathrm{BP}}^{t h}(\gamma)$ to determine the temperature dependence of $\gamma$ from the experimental values of $\omega_{\mathrm{BP}}(T) / \omega_{D}$. The resulting $\gamma / \gamma_{c}$ is reported in Fig. 5 (full line). A broadening of less than $4 \%$ of the elastic constants distribution, as indicated by the increase of $\gamma$, is sufficient to account for the strong reduction of the BP frequency. A corresponding variation is observed in the quantity $1 / Q_{1}^{3}$, the inverse of the cube of the FSDP position, as shown in Fig. 5 (open diamonds). Consequently, it appears that the broadening of the elastic constants distribution is induced by an increase of the local volume, proportional to $1 / Q_{1}^{3}$, confirming the strict relationship between the variation of the elastic properties and the evolution of the structure. 


\section{CONCLUSIONS}

In summary, we have investigated the evolution of the structure and of the vibrational dynamics of vitreous $\mathrm{GeO}_{2}$ over a wide temperature range, from the deep glassy state up to the supercooled liquid region. The BP frequency shows a marked softening as the temperature is increased above $T_{g}$. The temperature dependence of $\omega_{\mathrm{BP}}(T)$ is well described using a mean field approach, with a critical temperature $T_{\mathrm{BP}}$ $\simeq 1020 \mathrm{~K}$, intermediate between $T_{g}$ and $T_{m}$. The softening of the $\omega_{\mathrm{BP}}$ suggests that the evolution from the completely arrested phase to the supercooled liquid is accompanied by a modification of the dynamics of the system which seems to be governed by a process similar to a phase transition.

The temperature dependence of $\omega_{\mathrm{BP}}$ below $T_{g}$ is in good agreement with the variation of the Debye frequency. This observation indicates the BP change in the glass phase to be due to the temperature evolution of the macroscopic continuum medium. On the contrary, above $T_{g}$ the BP frequency experiences a strong variation, which is not observed for the Debye frequency. Both the longitudinal and transverse sound velocities and the density are, in fact, almost constant across $T_{g}$. The $\omega_{\mathrm{BP}}$ variation is accompanied by a small $(\sim 1 \%)$ reduction of the FSDP position. This small structural variation can account for the softening of the BP modes if it is accompanied by a broadening of the distribution of elastic constants between neighboring atoms.

We find a quantitative agreement between the evolution of the structure and the BP variation in the framework of the Schirmacher and co-workers model. ${ }^{10-12}$ The disorder parameter, $\gamma$, of the model increases with temperature, with a relative variation comparable to that of the local volume of the structure. This trend indicates a broadening of the distribution of elastic constants of the material as a consequence of the structural rearrangement. Such a variation of the force constant distribution does not affect the Debye frequency, which is determined from average values. On the contrary, in the low temperature region, where $\gamma$ is almost constant, the variation of the Debye frequency adequately describes the BP evolution. In the supercooled liquid the structure rapidly evolves towards a situation with increased shear stress fluctuations. This evolution is also influencing the dynamics which evolves into the non-Debye behavior.

\section{ACKNOWLEDGMENTS}

We acknowledge R. Dal Maschio for the preparation of our samples. The experiment at LLB was supported by the European Commission under the Sixth Framework Programme (Contract No. Rl13-CT-2003-505925).

${ }^{1}$ E. Fabiani, A. Fontana, and U. Buchenau, J. Chem. Phys. 128, 244507 (2008).

${ }^{2}$ U. Buchenau, A. Wischnewski, M. Ohl, and E. Fabiani, J. Phys. Condens. Matter 19, 205106 (2007).

${ }^{3}$ W. Doster, S. Cusack, and W. Petry, Nature (London) 337, 754 (1989).

${ }^{4}$ L. Hong, B. Begen, A. Kisliuk, C. Alba-Simionesco, V. N. Novikov, and A. P. Sokolov, Phys. Rev. B 78, 134201 (2008).

${ }^{5}$ U. Buchenau, N. Nücker, and A. J. Dianoux, Phys. Rev. Lett. 53, 2316 (1984).

${ }^{6}$ B. Rufflé, D. A. Parshin, E. Courtens, and R. Vacher, Phys. Rev. Lett. 100, 015501 (2008), and references therein.
${ }^{7}$ W. Götze and M. R. Mayr, Phys. Rev. E 61, 587 (2000).

${ }^{8}$ S. Taraskin, Y. L. Loh, G. Natarajan, and S. R. Elliott, Phys. Rev. Lett. 86, 1255 (2001).

${ }^{9}$ T. S. Grigera, V. Martin-Mayor, G. Parisi, and P. Verrocchio, Nature (London) 422, 289 (2003).

${ }^{10}$ W. Schirmacher, Europhys. Lett. 73(6), 892 (2006).

${ }^{11}$ W. Schirmacher, G. Ruocco, and T. Scopigno, Phys. Rev. Lett. 98, 025501 (2007).

${ }^{12}$ W. Schirmacher, B. Schmid, C. Tomaras, G. Viliani, G. Baldi, G. Ruocco, and T. Scopigno, Phys. Status Solidi 5, 862 (2008).

${ }^{13}$ E. Duval, A. Mermet, and L. Saviot, Phys. Rev. B. 75, 024201 (2007).

${ }^{14}$ H. Shintani and H. Tanaka, Nat. Mater. 7, 870 (2008).

${ }^{15}$ L. E. Silbert, A. J. Liu, and S. R. Nagel, Phys. Rev. Lett. 95, 098301 (2005).

${ }^{16}$ N. Xu, V. Vitelli, A. J. Liu, and S. R. Nagel, Europhys. Lett. 90, 56001 (2010).

${ }^{17}$ K. Chen, W. G. Ellenbroek, Z. Zhang, D. T. N. Chen, P. J. Yunker, S. Henkes, C. Brito, O. Dauchot, W. van Saarloos, A. J. Liu, and A. G. Yodh, Phys. Rev. Lett. 105, 025501 (2010).

${ }^{18}$ O. Pilla, L. Angelani, A. Fontana, J. R. Gonsalves, and G. Ruocco, J. Phys. Condens. Matter 15, S995 (2003).

${ }^{19}$ O. Pilla, S. Caponi, A. Fontana, J. R. Gonsalves, M. Montagna, F. Rossi, G. Viliani, L. Angelani, G. Ruocco, G. Monaco, and F. Sette, J. Phys. Condens. Matter 16, 8519 (2004).

${ }^{20}$ B. Ruzicka, T. Scopigno, S. Caponi, A. Fontana, O. Pilla, P. Giura, G. Monaco, E. Pontecorvo, G. Ruocco, and F. Sette, Phys. Rev. B 69, 100201 (2004).

${ }^{21}$ G. Baldi, V. M. Giordano, G. Monaco, F. Sette, E. Fabiani, A. Fontana, and G. Ruocco, Phys. Rev. B 77, 214309 (2008).

${ }^{22}$ L. Orsingher, G. Baldi, A. Fontana, L. Bove, T. Unruh, A. Orecchini, N. Violini, C. Petrillo, and F. Sacchetti, Phys. Rev. B 82, 115201 (2010).

${ }^{23}$ G. Baldi, V. Giordano, G. Monaco, and B. Ruta, Phys. Rev. Lett 104, 195501 (2010).

${ }^{24}$ A. I. Chumakov, G. Monaco, A. Monaco, W. A. Crichton, A. Bosak, R. Rüffer, A. Mayer, F. Kargl, L. Comez, D. Fioretto, H. Giefers, S. Roitsch, G. Wortmann, M. H. Manghnani, A. Hashur, Q. Williams, J. Balogh, K. Parliński, P. Jochym, and P. Piekarz, Phys. Rev. Lett. 106, 225501 (2011)

${ }^{25}$ S. Caponi, A. Fontana, F. Rossi, G. Baldi, and E. Fabiani, Phys. Rev. B 76, 092201 (2007).

${ }^{26}$ G. Baldi, A. Fontana, G. Monaco, L. Orsingher, S. Rols, F. Rossi, and B. Ruta, Phys. Rev. Lett. 102, 195502 (2009).

${ }^{27}$ B. Rufflé, S. Ayrinhac, E. Courtens, R. Vacher, M. Foret, A. Wischnewski, and U. Buchenau, Phys. Rev. Lett. 104, 067402 (2010).

${ }^{28}$ K. Niss, B. Begen, B. Frick, J. Ollivier, A. Beraud, A. P. Sokolov, V. N. Novikov, and C. Alba-Simionesco, Phys. Rev. Lett. 99, 055502 (2007).

${ }^{29}$ T. Deschamps, C. Martinet, D. R. Neuville, D. de Ligny, C. Coussa-Simon, and B. Champagnon, J. Non-Cryst. Solids 355, 2422 (2009).

${ }^{30}$ T. Deschamps, C. Martinet, D. de Ligny, J. L. Bruneel, and B. Champagnon, J. Chem. Phys. 134, 234503 (2011).

${ }^{31}$ A. Monaco, A. I. Chumakov, G. Monaco, W. A. Crichton, A. Meyer, L. Comez, D. Fioretto, J. Korecki, and R. Rüffer, Phys. Rev. Lett. 97, 135501 (2006).

${ }^{32}$ L. Orsingher, A. Fontana, E. Gilioli, G. Carini, Jr., G. Carini, G. Tripodo, T. Unruh, and U. Buchenau, J. Chem. Phys. 132, 124508 (2010).

${ }^{33}$ M. Zanatta, G. Baldi, S. Caponi, A. Fontana, E. Gilioli, M. Krish, C. Masciovecchio, G. Monaco, L. Orsingher, F. Rossi, G. Ruocco, and R. Verbeni, Phys. Rev. B 81, 212201 (2010).

${ }^{34}$ S. Caponi, S. Corezzi, D. Fioretto, A. Fontana, G. Monaco, and F. Rossi, Phys. Rev. Lett. 102, 027402 (2009).

${ }^{35}$ J. Hertling, S. Baessler, S. Rau, G. Kasper, and S. Hunklinger, J. Non-Cryst. Solids 226, 129 (1998).

${ }^{36}$ A. V. Ananev, V. N. Bogdanov, B. Champagnon, M. Ferrari, G. O. Karapetyan, L. V. Maksimov, S. N. Smerdin, and V. A. Solovyev, J. Non-Cryst. Solids 354, 3049 (2008).

${ }^{37}$ F. Rossi, G. Baldi, S. Caponi, R. Dal Maschio, A. Fontana, L. Orsingher, and M. Zanatta, Philos. Mag. 91, 1910 (2011).

${ }^{38}$ E. F. Riebling, J. Chem. Phys. 39, 3022 (1963).

${ }^{39}$ K. Kamiya, T. Yoko, Y. Itoh, and S. Sakka, J. Non-Cryst. Solids 79, 285 (1986).

${ }^{40}$ B. Schmid and W. Schirmacher, Phys. Rev. Lett. 100, 137402 (2008).

${ }^{41}$ F. L. Galeener and P. N. Sen, Phys. Rev. B 17, 1928 (1978).

${ }^{42}$ C. Petrillo and F. Sacchetti, Acta Crystallogr., Sect. A: Found. Crystallogr. 46, 440 (1990). 
${ }^{43}$ C. Petrillo and F. Sacchetti, Acta Crystallogr., Sect. A: Found. Crystallogr. 48, 508 (1992).

${ }^{44}$ S. R. Elliot, Nature (London) 354, 445 (1991).

${ }^{45}$ P. S. Salmon, A. C. Barnes, R. A. Martin, and G. J. Cuello, Phys. Rev. Lett. 96, 235502 (2006).

${ }^{46}$ A. P. Sokolov, A. Kisliuk, M. Soltwisch, and D. Quitmann, Phys. Rev. Lett. 69, 1540 (1992).

${ }^{47}$ A. Fontana, R. DellAnna, M. Montagna, F. Rossi, G. Viliani, G. Ruocco, M. Sampoli, U. Buchenau, and A. Wischnewski, Europhys. Lett. 47, 56 (1999).
${ }^{48}$ A. Fontana, F. Rossi, G. Viliani, S. Caponi, E. Fabiani, G. Baldi, G. Ruocco, and R. Dal Maschio, J. Phys. Condens. Matter 19, 205145 (2007).

${ }^{49}$ A. P. Sokolov, U. Buchenau, W. Steffen, B. Frick, and A. Wischnewski, Phys. Rev. B 52, R9815 (1995).

${ }^{50}$ N. V. Surovtsev and A. P. Sokolov, Phys. Rev. B 66, 054205 (2002).

${ }^{51}$ K. Huang, Statistical Mechanics, 2nd ed. (Wiley, New York, 1987).

${ }^{52}$ T. Scopigno, M. Dastuto, M. Krisch, G. Ruocco, and F. Sette, Phys. Rev. B 64, 012301 (2001). 